\title{
The Application of the Recent Advances in Stereophotogrammetry for the Diagnosis and Management of Oro-Facial Deformities
}

\author{
Balvinder S. KHAMBAY*a, Xiangyang $\mathrm{JU}^{\mathrm{b}}$, Thamer AL-ANEZI ${ }^{\mathrm{a}}$, Ashraf AYOUB $^{\mathrm{a}}$ \\ ${ }^{a}$ Biotechnology and Craniofacial Sciences Research Group, Glasgow University, UK \\ ${ }^{b}$ Department of Clinical Physics, NHS Greater Glasgow and Clyde, UK
}

\begin{abstract}
This presentation will illustrate the recent innovations in 3D stereophotogrammetry to provide a useful technique for accurate recording of the face dysmorphology in children with cleft lip and palate and patients with facial deformities who have had orthognathic surgery. Using a custom developed software program facial landmarks were digitised on the computerised 3D model, Procrustes analysis was applied to superimpose the 3D co-ordinates of anatomical landmarks at different time intervals. Principal component statistical analysis was applied to assess the magnitude of surgical changes. The method was accurate to within $0.5 \mathrm{~mm}$ and has been shown to be reliable in infants. Significant soft tissue changes were detected following orthognathic surgery; these were stable at 6 months following surgery when compared with the immediate postoperative appearance. The validity of automatic tracking of anatomical landmarks during facial animations has also been investigated using Di4D software. This presentation will highlight the clinical applications of 4D imaging and debate the accuracy of 3D prediction planning for correction of dentofacial deformities.
\end{abstract}

Keywords: 3d body scanning, proceedings

\section{Introduction}

Three-dimensional imaging techniques have attempted to overcome the shortcomings of conventional two-dimensional methods [1-6]. These techniques have included: morphanalysis [7], laser scanning $[8,9], 3 D$ computerized tomography scanning [10], Stereolithography [11], 3D ultrasonography [12], 3D facial morphometry [13,14], digigraph imaging [15], Moiré topography [16] and contour photography [17].

Stereophotogrammetry is a technique of facial imaging by using two cameras, configured as a stereopair, to generate the 3D configuration of the face by triangulation. To reduce inaccuracy due to possible head movement and change in facial expression, images from each side of the face are taken simultaneously and the duration of exposure has been reduced with improvement in technology [18]. A vision-based non-contact 3D imaging system (C3D®) has been developed at Glasgow University to be utilised for clinical assessment of facial morphology $[19,20]$ The system is described in more detail elsewhere [21]. Its capture time is one milliseconds and its accuracy is within $0.26 \mathrm{~mm}$ in the three directions [22,23]. Since its introduction the technology is now commercially available.

\section{Aims}

The aim of this presentation is to explore the broad application of 3D imaging in quantifying facial deformities and assessing surgical outcomes in the following groups of patients:

- Following surgical repair of cleft lip \& palate.

- Patients who had had surgical correction of dento-facial deformities

- $\quad$ The validation and future applications of 3D motion capture.

\section{Cleft lip \& palate}

A study to evaluate three-dimensional lip morphology, following primary reconstruction in children with unilateral cleft lip and palate relative to contemporaneous non-cleft data was carried out at the Glasgow Dental School, University of Glasgow [24].

*Balvinder.Khambay@glasgow.ac.uk 
Two groups of 3-year-old children were recruited (21 with unilateral cleft lip and palate and 96 controls) facial images were taken using a three-dimensional vision-based capture technique.

The three-dimensional images of the face were reflected so the cleft was on the left side to create a homogeneous group for statistical analysis. Three-dimensional coordinates of anthropometric landmarks were extracted from the facial images. Three-dimensional, generalized Procrustes superimposition was implemented and a set of linear measurements were used to compare cleft and control subjects for right and left sides, adjusting for sex differences.

The results showed that crista philtri on both the cleft and non-cleft sides were displaced laterally and posteriorly; there was also a statistically significant increase in philtrum width. No significant differences between cleft and control regarding the cutaneous height of the upper lip. The lip in the cleft patients was flatter than in the non-cleft individuals, with less prominence of labialis superioris.

In conclusion, stereophotogrammetry allows detection of residual dysmorphology following cleft repair. There was significant increase of the philtrum width. The lip appeared flatter and more posterior displaced in unilateral cleft lip and palate patients compared with controls.

In a further study the group evaluated the three-dimensional (3D) lip morphology following primary reconstruction in children with unilateral cleft lip and palate (UCLP) relative to contemporaneous noncleft data [25].

Three groups of 10-year-old children: 51 with UCLP, 43 UCL (unilateral cleft lip), and 68 controls.

Three-dimensional images of the face were recorded using stereo cameras on a two-pod capture station, and 3D coordinates of anthropometric landmarks were extracted from the facial images. A novel method was applied to quantify residual lip dysmorphologies using Principle Component Analysis (PCA).

Residual lip dysmorphologies were more pronounced in UCLP cases. The width of the Cupid's bow was increased due to lateral displacement of the christa philteri left (cphL) in both UCL and UCLP patients. In the upper part of the lip, the nostril base was significantly wider in UCLP cases when compared with UCL cases and controls.

In conclusion stereophotogrammetry, together with associated image analysis, allows early detection of residual dysmorphology following cleft repair.

\section{Orthognathic Surgery}

3D facial images were taken using a stereophotogrammetry-based 3D imaging system (Di3D® system) before and after surgery for 44 patients: 20 Class III cases treated by bimaxillary osteotomy; 12 Class III cases treated by maxillary advancement alone, and 12 Class II cases treated by bimaxillary operations. 3D images were taken within a week before operation (T1), and one (T2), three (T3), and six (T4) months after operation [26].

3D facial models were exported as Virtual Reality Modelling Language (VRML) models to be inserted into the Facial Analysis Tool $囚$ (FAT). Each 3D model was loaded and displayed in 3 viewing windows. The landmarks were directly digitised on the 3D model on the screen. A text file containing $x, y$ and $z$ coordinates of each landmark was produced and saved for analysis.

The original configuration of landmarks for each patient at each assessment time was scaled to a common size. Each of the configurations was reflected around an arbitrary plane and a mirror image (reflected) configuration was generated. The original configuration was superimposed on the reflected configuration using partial Ordinary Procrustes Analysis (OPA). The partial Ordinary Procrustes superimposition involved two steps: translation and rotation. First, the geometric centres of the two configurations (original and reflected) were found. Then they were translated so that they were superimposed on their geometric centre. Rotation followed the translation, moving one configuration about its geometric centre until the best fit was found between all homologous landmarks while preserving the information about shape and size of each configuration. The 'individual symmetrical configuration' was created by calculating the mean of the original configuration and the reflected version after aligning. This was followed by superimposition of the original configuration on the created individual symmetrical configuration using partial OPA again.

The mean squared distances between landmarks in the original configurations and their corresponding landmarks in the individual symmetrical configurations were expressed as a general facial asymmetry score for each subject. The perfect symmetrical face would have a score of zero. The higher the score, the more asymmetrical the face. 
The values presented in the 3D asymmetry analysis were stated in terms of units. Facial landmarks configurations were scaled in order to allow comparisons regardless of size differences between configurations. The use of units instead of millimetres might be considered a drawback. However, the advantage of the current analysis lies in its independence of any assumed symmetry plane to reflect (or mirror) soft-tissue landmarks as well as lack of influence of size on the calculated asymmetry scores. In addition, the analysis is not affected by the direction of asymmetry. In other words, patients with left deviations of the chin can be included in the same sample of patients with right deviations of the chin without the concern of positive differences cancelling out negative differences. The landmarks employed in the current analysis have been found to be highly reproducible in a previous investigation [21] with an error below $0.5 \mathrm{~mm}$ in landmark identification. Any inconsistencies in landmark identification above this limit may reduce the diagnostic value and sensitivity of such analyses.

In the bimaxillary osteotomy group, facial symmetry improved after operation. In the maxillary advancement group, there was no notable improvement in facial symmetry after the operation. In the bimaxillary group, facial symmetry deteriorated after the operation, particularly at the tip of the nose and prominence of the chin. At six months follow-up, the changes in facial asymmetry in the three groups were not significant.

\section{3D motion analysis}

A paralysis or weakness of one side of the face can significantly impact an individual's quality of life and appearance. The patient often has difficulty performing basic daily functions such as eating, drinking, and swallowing and communicating. There is a known increased risk for moderate to serious levels of depression and anxiety in such patients. In some situations the nerve supplying the facial muscles may have had to be surgically removed and may require repair. Techniques aimed at correcting the paralysis include nerve "replacement" surgery, trying to join the ends of the cut nerve together or muscle and nerve transfer.

Important treatment goals for a patient with facial paralysis are to achieve facial symmetry at rest and to return the ability to smile. However, there are no widely accepted measurement techniques for the assessment of these goals. The present methods either involve describing the loss of function in words, taking measurements directly from the patient's face using a ruler, or measurements from a conventional photograph or video [27]. These techniques are not very reproducible and are time consuming. As a result, it is difficult to measure the effectiveness of a medical intervention or a surgical reconstruction. A usable measurement system would allow comparison of preoperative and postoperative facial position and movement. By comparing the paralyzed side with the normal side, the degree of deformity and the effectiveness of surgery could be identified.

Advances in 3-dimensional data capture, three dimensional motion tracking, and computer modeling now allow for more appropriate measurement and analysis of facial animation. Colour 3D motion capture video systems have been developed to capture colour 3D motion stereo video sequences of a face. The system in current use (Di4D, Dimensional Imaging, Glasgow, U.K.) also has a unique function in which the first image of the system only needs to be landmarked and the remaining sequence is automatically landmarked, reducing the time and errors involved in manual landmark placement.

Research in facial paralysis outcomes has traditionally been evaluated with subjective measures. Current 4D methods are far superior and generate reproducible, accurate, and objective data for such clinical studies. As these technologies become more readily available, we would anticipate a paradigm shift in how this type of research is conducted.

\section{References}

1. Proffit WR and Turvey TA. Dentofacial Asymmetry. In: Proffit WR and White RP, Jr. eds. Surgical Orthodontic Treatment. St. Louis: Mosby - Year Book, Inc., 1991: 483-549.

2. Ferrario VF, Sforza C, Poggio CE, Tartaglia G. Distance from symmetry: a three-dimensional evaluation of facial asymmetry. J Oral Maxillofac Surg 1994; 52: 1126-1132.

3. Garrahy A. 3D assessment of cleft and non-cleft children. PhD thesis, University of Glasgow, 2002.

4. McIntyre GT and Mossey PA. Asymmetry of the parental craniofacial skeleton in orofacial clefting. J Orthod 2002; 29: 299-305. 
5. McIntyre GT and Mossey PA. Posteroanterior cephalometric analysis of the parental craniofacial morphology in orofacial clefting. Cleft Palate Craniofac J 2003; 40: 416-425.

6. Ayoub AF, Wray D, Moos KF, Siebert P, Jin J, Niblett TB, Urquhart C, Mowforth R. Threedimensional modeling for modern diagnosis and planning in maxillofacial surgery. Int $\mathrm{J}$ Adult Orthodon Orthognath Surg 1996; 11: 225-233.

7. Rabey G. Craniofacial morphanalysis. Proc R Soc Med 1971; 64: 103-111.

8. McCance AM, Moss JP, Wright WR, Linney AD, James DR. A three-dimensional soft tissue analysis of 16 skeletal class III patients following bimaxillary surgery. $\mathrm{Br} \mathrm{J}$ Oral Maxillofac Surg 1992; 30: 221-232.

9. Moss JP, McCance AM, Fright WR, Linney AD, James DR. A three-dimensional soft tissue analysis of fifteen patients with Class II, Division 1 malocclusions after bimaxillary surgery. Am J Orthod Dentofacial Orthop 1994; 105: 430-437.

10. McCance AM, Moss JP, Fright WR, James DR, Linney AD. A three dimensional analysis of soft and hard tissue changes following bimaxillary orthognathic surgery in skeletal III patients. $\mathrm{Br} \mathrm{J}$ Oral Maxillofac Surg 1992; 30: 305-312.

11. Bill JS, Reuther JF, Dittmann W, Kubler N, Meier JL, Pistner H, Wittenberg G. Stereolithography in oral and maxillofacial operation planning. Int J Oral Maxillofac Surg 1995; 24: 98-103.

12. Hell B. 3D sonography. Int J Oral Maxillofac Surg 1995; 24: 84-89.

13. Ferrario VF, Sforza C, Serrao G, Puletto S, Bignotto M, Tartaglia G. Comparison of soft tissue facial morphometry in children with Class I and Class II occlusions. Int J Adult Orthodon Orthognath Surg 1994; 9: 187-194.

14. Ferrario VF, Sforza C, Poggio CE, Serrao G, Miani A, Jr. A three-dimensional study of sexual dimorphism in the human face. Int J Adult Orthodon Orthognath Surg 1994; 9: 303-310.

15. Nanda RS, Ghosh J, Bazakidou E. Three-dimensional facial analysis using a video imaging system. Angle Orthod 1996; 66: 181-188.

16. Kawai $\mathrm{T}$, Natsume N, Shibata $\mathrm{H}$, Yamamoto $\mathrm{T}$. Three-dimensional analysis of facial morphology using moire stripes. Part I. Method. Int J Oral Maxillofac Surg 1990; 19: 356-358.

17. Leivesley WD. The reliability of contour photography for facial measurements. $\mathrm{Br} \mathrm{J}$ Orthod 1983; 10: 34-37.

18. Siebert JP and Marshall S. Human body 3D imaging by speckle texture projection photogrammetry. Sensor Review 2000; 20: 218-226.

19. Ayoub AF, Wray D, Moos KF, Jin J, Niblett TB, Urquhart C, Mowforth P, Siebert P. A threedimensional imaging system for archiving dental study casts: a preliminary report. Int $\mathrm{J}$ Adult Orthodon Orthognath Surg 1997; 12: 79-84.

20. Ayoub AF, Siebert P, Moos KF, Wray D, Urquhart C, Niblett TB. A vision-based threedimensional capture system for maxillofacial assessment and surgical planning. $\mathrm{Br} \mathrm{J}$ Oral Maxillofac Surg 1998; 36: 353-357.

21. Hajeer MY, Ayoub AF, Millett DT, Bock M, Siebert JP. Three-dimensional imaging in orthognathic surgery - the clinical application of a new method. Int J Adult Orthodon Orthognath Surg 2002; 17: 318-330.

22. Hajeer MY. 3D Soft-Tissue, 2D Hard-Tissue and Psychosocial Changes Following Orthognathic Surgery. Ph.D thesis, University of Glasgow, 2003.

23. A Ayoub, A Garrahy, C Hood, J White, M Bock, J P Siebert, R Spencer, A Ray. Validation of a vision based three-dimensional facial imaging system. Cleft Palate Craniofac J 40:523-529, 2003

24. Ayoub A, Garrahy A, Millett D, Bowman A, Siebert JP, Miller J, Ray A.

Three-dimensional assessment of early surgical outcome in repaired unilateral cleft lip and palate: part 2. Lip changes. Cleft Palate Craniofac J. 2011; 48(5):578-83.

25. Ayoub A, Bell A, Simmons D, Bowman A, Brown D, Lo TW, Xiao Y. 3D Assessment of Lip Scarring and Residual Dysmorphology Following Surgical Repair of Cleft Lip and Palate: A Preliminary Study. Cleft Palate Craniofac J. 2011; 48(4):379-87.

26. Hajeer MY, Ayoub AF, Millett DT. Three-dimensional assessment of facial soft-tissue asymmetry before and after orthognathic surgery. Br J Oral Maxillofac Surg. 2004; 42(5):396-404.

27. Burres $S$, Fisch $U$. The comparison of facial grading systems. Arch Otolaryngol Head Neck Surg. 1986, (7):755-758. 